\title{
Carbonatación de diatomeas y génesis de calizas/ dolomías diagenéticas en ambiente continental y marino (Mioceno de Tresjuncos, Cuenca y Níjar, Almería). Un ejemplo de secuestro de $\mathrm{CO}_{2}$ en la Naturaleza
}

\author{
Carbonatation of diatomites and diagenetic limestones/ \\ dolomites in freshwater and marine environments (Miocene of \\ Tresjuncos, Cuenca and Nijar, Almería). An example of $\mathrm{CO}_{2}$ \\ sequestration in Nature
}

\author{
M.A. Bustillo ${ }^{1}$ \\ ${ }^{1}$ Departamento de Geología. Museo Nacional de Ciencias Naturales, CSIC. C/ José Gutiérrez Abascal 2, 28006. Email: \\ abustillo@mncn.csic.es; ORCID ID: https://orcid.org/0000-0003-3626-4609
}

\section{RESUMEN}

En el presente trabajo se estudian los procesos de carbonatación de frústulas de diatomeas y la consecuente formación de calizas/dolomías diagenéticas, en estratos de diatomitas marinas (Messiniense, sección de Níjar) y lacustres (Turoliense, sección de Tresjuncos). Se realiza un estudio mineralógico y petrológico para conocer el reemplazo de ópalo A por calcita o dolomita, considerando los factores que favorecen este reemplazo en ambos ambientes. Los datos obtenidos son relevantes para la investigación del uso de diatomitas en la captura y almacenamiento de dióxido de carbono a través de su carbonatación.

Los procesos pseudomórficos de carbonatación de las valvas se llevaron a cabo mediante el reemplazo directo del ópalo A por calcita o dolomita y la cementación de la microporosidad. La perfecta reproducción de las valvas indica un mecanismo de reacción con acoplamiento de disolución-precipitación en la interfase de reacción. Se interpreta que la organización de las microesferas de ópalo A, dentro de las valvas, condicionaría la organización de complejos amorfos de varios iones o nanopartículas de carbonatos amorfos, que posteriormente recristalizarían a calcita o dolomita.

En las diatomitas lacustres, el proceso de carbonatación de las valvas fue favorecido por la materia orgánica proveniente de la gran cantidad de biota (anfibios, crustáceos, insectos, plantas... etc.) que incorporan. En este ambiente, los procesos de sulfato-reducción durante la degradación de la materia orgánica, generaron $\mathrm{CO}_{2}$, que junto con cambios de $\mathrm{pH}$ y salinidad facilitaron los reemplazos. Es posible que las carbonataciones de las valvas fueran coetáneas a la precipitación de calcita y dolomita que fosiliza la biota y por lo tanto, diagenéticas tempranas. En las diatomitas marinas, el proceso desencadenante del reemplazo de las valvas fue la formación de capas lenticulares y nódulos opalinos, ya que al disolverse los microfósiles calcáreos que las diatomitas incluían, se liberó $\mathrm{CO}_{2}$ y Ca. Pequeñas variaciones de $\mathrm{pH}$ en un entorno alrededor de 9 , y la presencia de sulfatos facilitarían el reemplazado durante la diagénesis por enterramiento.

Palabras clave: carbonatación; diatomitas; ópalo A; pseudomorfismo; diagénesis.

Citation / Cómo citar este artículo: Bustillo, M.A. (2020). Carbonatación de diatomeas y génesis de calizas/dolomías diagenéticas en ambiente continental y marino (Mioceno de Tresjuncos, Cuenca y Nijar, Almería). Un ejemplo de secuestro de $\mathrm{CO}_{2}$ en la Naturaleza. Estudios Geológicos 76(1): e125. https://doi.org/10.3989/egeol.43607.541.

Copyright: () 2020 CSIC. This is an open-access article distributed under the terms of the Creative Commons Attribution-Non Commercial (by-nc) Spain 4.0 License. 


\begin{abstract}
Carbonate (calcite and dolomite) pseudomorphs after diatoms were identified from marine (Messinian, Níjar section) and freshwater (Turolian, Tresjuncos section) diatomites. The mineralogical and petrological study has allowed to know the replacement of opal-A by calcite or dolomite, and the consequent formation of diagenetic limestones/dolostones, deducing the factors that favor this replacement in both environments. The data obtained are relevant to the research about the use of diatomites for the capture and storage of carbon dioxide through carbonation.

A pseudomorphic carbonatation process of the frustules occurred by a direct replacement of the opal-A and a cementation of the microporosity. Opal-A was replaced by calcite or dolomite via a coupled dissolution-precipitation process. The organization of the opal A microspheres inside the frustules could determine the organization of multiion complexes / nanoparticles of amorphous carbonates that would later recrystallize to calcite or dolomite crystals.

The carbonation of the lacustrine diatomites was favored by the great amount of biota (amphibians, crustaceans, insects, plants...etc.) included in them. In this environment, the sulphate-reduction processes during the degradation of the organic matter generated $\mathrm{CO}_{2}$, which along with $\mathrm{pH}$ and salinity changes, facilitated the replacements. It is possible that the carbonatation of the frustules was coeval to the calcite or dolomite precipitation during the fossilization of the biota, and therefore, early diagenetic in origin.

The nodules and opaline lenticular beds formation triggered the carbonation process of the marine diatomites because $\mathrm{CO}_{2}$ and $\mathrm{Ca}$ are released from the dissolution of biocalcarenites/biocalcitutites included in the diatomites. Small $\mathrm{pH}$ variations around 9, and the presence of sulfates, would facilitate the replacement of the valves, during the burial diagenesis.
\end{abstract}

Keywords: carbonatation; diatomites; opal-A; pseudomorphism; diagenesis.

\section{Introducción}

Una gran mayoría de las calizas de series marinas y continentales son depósitos biogénicos formados por la acumulación de organismos calcáreos y por procesos de cementación y recristalización a partir de ellos. Calcita biogénica y aragonito son frecuentemente los puntos de partida de transformaciones que generan calizas litificadas y dolomías. Sin embargo, diatomitas, marinas o continentales, pueden ser punto de partida de calizas o dolomías diagenéticas por procesos de carbonatación de diatomeas. La calcificación de diatomeas ha sido descrita puntualmente tanto en ambiente continental (Calvo et al., 1988, Bellanca et al., 1989, Bustillo et al., 2017b) como marino (Hein et al., 1979, 1990), pero sólo Hein et al. (1979) establece una relación entre este proceso y la formación de calizas diagenéticas. Los procesos de silicificación de carbonatos son ampliamente conocidos y descritos en la literatura científica (Liesegang, 2017), sin embargo los procesos inversos de carbonatación de depósitos silíceos son mucho menos reconocidos y estudiados.

Es objetivo de este trabajo el estudio de los procesos de carbonatación de diatomeas en dos ambientes muy diferentes como son el ambiente continental diagenético temprano y poco profundo (sección de Tresjuncos, Fig.1) y el ambiente diagenético marino por enterramiento (sección de Níjar Fig.1). Se pretende comprender el proceso del reemplazo del ópalo A de las valvas por calcita o dolomita, y a su vez determinar si hay factores comunes que favorecen dicho reemplazo en ambientes diferentes. Conocer como mineralizar el $\mathrm{CO}_{2}$, para optimizar las condiciones de secuestro del $\mathrm{CO}_{2}$ a través de la precipitación de carbonatos y otros procesos, ha sido motivo de estudio por varios autores en la última década (Flaathen et al., 2011, Bickle et al., 2017). En este sentido, la transformación de las valvas de diatomeas en calcita o dolomita es un proceso de carbonatación que puede servir de pauta para investigar el uso de diatomitas como almacén subterráneo de $\mathrm{CO}_{2}$. Martínez del Olmo (2019) incide en la importancia de determinar claramente las posibles trampas geológicas de $\mathrm{CO}_{2}$ en España, valorando diferentes zonas y formaciones geológicas. Los casos analizados se basan en el almacenaje de $\mathrm{CO}_{2}$ pero no en la posible fijación por precipitación de carbonatos.

\section{Zonas de estudio y contextos geológicos}

Los depósitos continentales escogidos se encuentran en la localidad de Tresjuncos (Fig. 1) (Cuenca) situada geológicamente en el interior del cinturón de pliegues y cabalgamientos de la Sierra de Altomira, que separa las cuencas cenozoicas de 


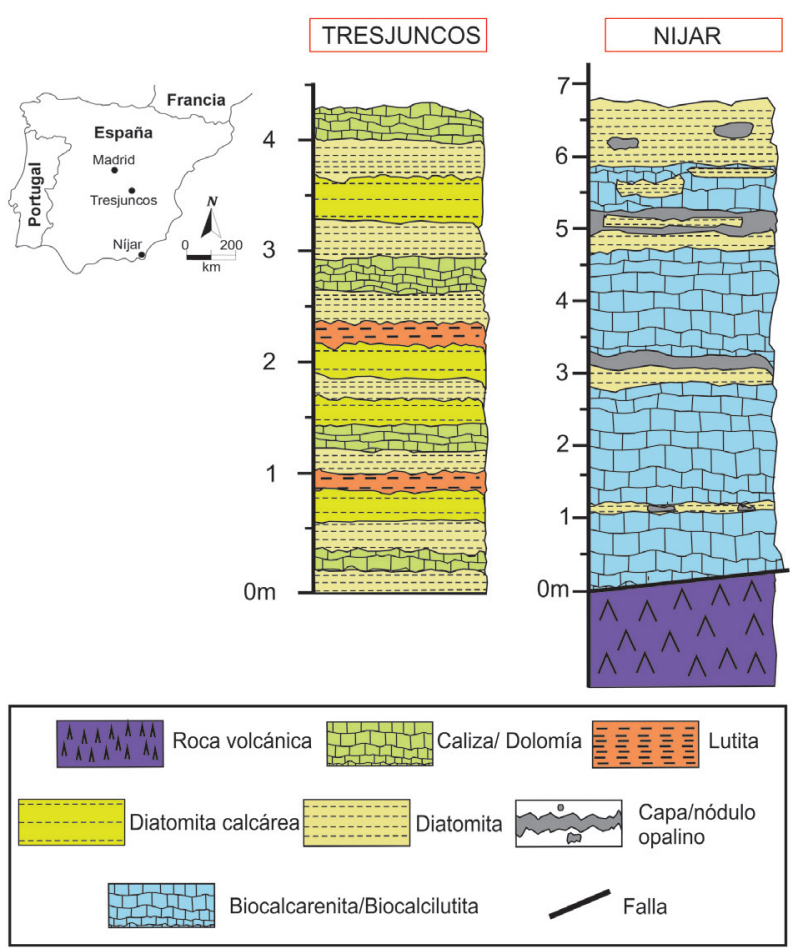

Figure 1.-Secciones litológicas estudiadas, y su localización.

Madrid y de Loranca. La sucesión estratigráfica en la que se hallan las muestras estudiadas forma parte de la Unidad V del relleno de la cuenca de Loranca. Esta unidad tiene una edad Vallesiense-Turoliense (Gómez et al., 1996; Bustillo et al., 2017b) y en ella se reconocen depósitos de abanicos aluviales (lutitas, brechas y conglomerados) que en sentido vertical pasan a margas y calizas de ambientes palustres y lacustres (Díaz- Molina \& Tortosa, 1996; Hernaíz \& Cabra, 1998).

El muestro en Tresjuncos fue realizado en Cerro Molino, en una sección de calizas, diatomitas calcáreas, diatomitas y lutitas (Figs.1 y 2A), definida como lacustre (Bustillo et al., 2017b). Según estos autores, esta sección pasa lateralmente y hacia arriba a calizas lacustres, definidas como wackestones/ packestones de ostrácodos que incorporan también gasterópodos, carofitas, pellets y pequeñas cantidades de cuarzo terrígeno. Los paleosuelos, localizados al norte de la sección, representarían la orla palustre del lago, donde se han interpretado fenómenos de hidromorfismo y calcificación de cristales de yeso. A su vez la disminución de carbonatos hacia el sur y el aumento del espesor de las diatomitas se relacionaron con un alejamiento del margen del lago y un ligero aumento de la profundidad. Es precisamente en esta zona algo más profunda donde se localizan las calizas y dolomías estudiadas que aparecen intercaladas con las diatomitas.

Las diatomitas lacustres tienen una laminación paralela submilimétrica, que muestra plegamientos y minifracturas, probablemente producidos por compactación. Sobre los planos de estratificación y entre las láminas son frecuentes los restos de artrópodos, insectos, vegetales, semillas.. etc. (Bustillo et al. 2017b).Pueden alternar con capas delgadas de lutitas grises formando secuencias rítmicas. Las calizas y dolomías forman capas que muchas veces reproducen las características de las diatomitas, de modo que reflejan sus finas laminaciones o las deformaciones dentro del estrato (Fig. 2B).

Las diatomitas marinas estudiadas (Fig.1, sección de Níjar) se hallan en la región volcánica del cabo de Gata y pertenecen a la formación descrita como silexitas, margas y turbiditas carbonatadas, de edad Messiniense (Pineda et al., 1981). Durante el Messiniense existe gran diversificación de facies por la paleogeografía compleja de corredores y plataformas marinas entre relieves volcánicos. Los sedimentos más profundos de estas cuencas son denominados, en general, margas e incluyen microorganismos plantónicos calcáreos y silíceos con predominio de diatomeas. La sucesión sedimentaria estudiada se localiza entre dos zonas de rocas volcánicas, con las que se halla en contacto por falla. Las muestras fueron obtenidas en un talud de la carretera 3108, entre las localidades de San Isidro de Níjar y San Jose (coordenadas: Lat.: $36^{\circ}$ $51^{\prime}$ y $50^{\prime \prime} \mathrm{N}$, Long.: $\left.-2^{\circ} 9^{\prime} 44^{\prime \prime} \mathrm{W}\right)$. Biocalcarenitas/ biocalcilutitas (tonos pardos, Fig. 2C) y diatomitas (tonos blancos, Fig. 2C) son las dos litologías esenciales que componen la sección aunque también aparecen litologías intermedias donde se intercalan láminas milimétricas de ambas. La sección incluye varias capas lenticulares y nódulos de ópalo que se localizan en los tramos diatomíticos. Alrededor de estas capas y nódulos, y a veces en su interior, pueden existir zonas claras que destacan por ser más compactas que las diatomitas (Figs. 2D y E) y que después de su estudio se ha determinado que son consecuencia de los procesos de carbonatación de diatomeas (Fig. 2F). 

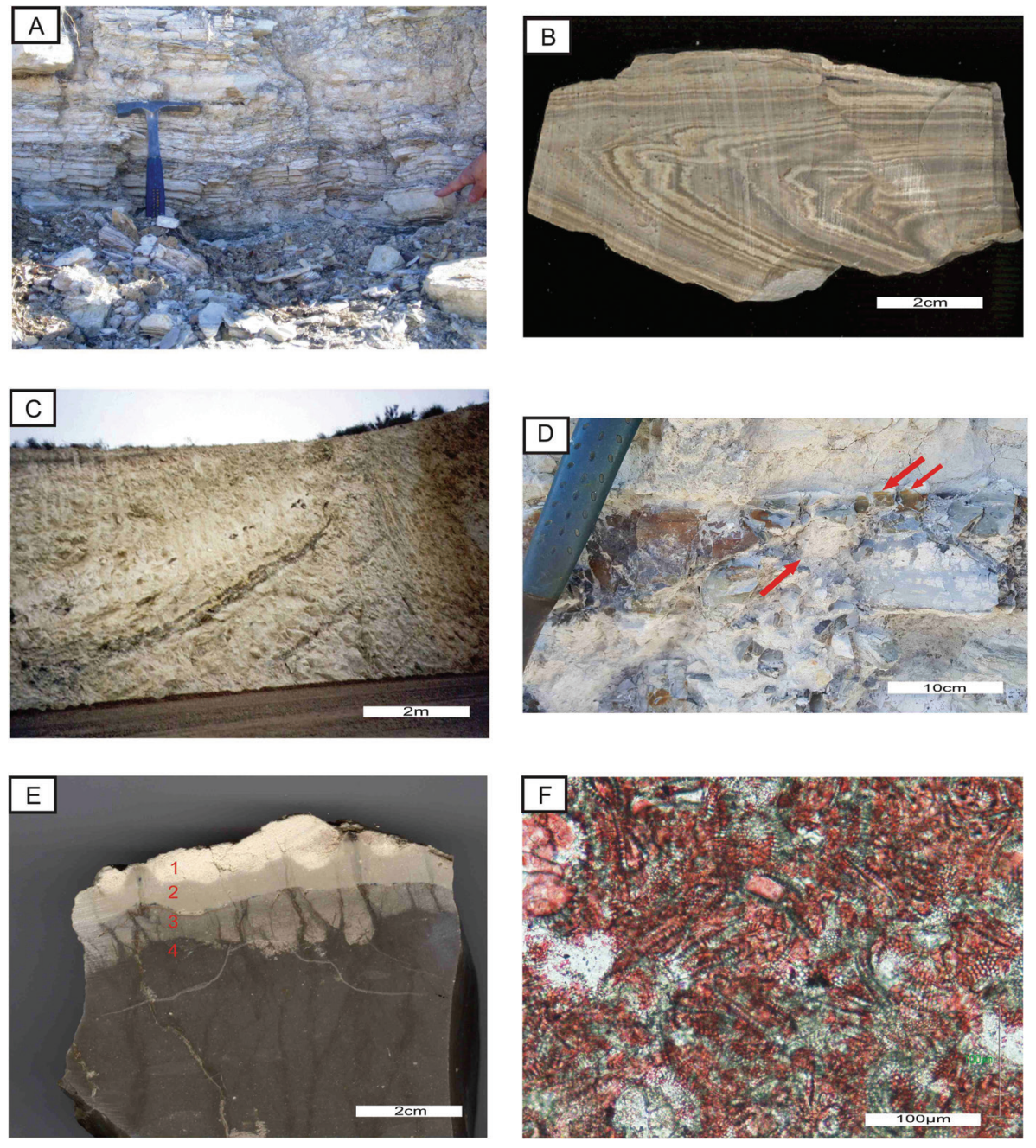

Figure 2.-A. Parte intermedia de la sección de Tresjuncos. Intercalaciones centimétricas de diatomitas, lutitas y calizas o dolomías diagenéticas; B. Detalle de una caliza diagenética de la sección anterior, donde queda preservada la primitiva laminación diatomítica replegada; C. Talud de carretera entre Níjar y San José. Intercalaciones métricas de diatomitas y biocalcarenitas/biocalcilutitas. En las diatomitas aparecen capas lenticulares y nódulos opalinos; D. Detalle de una capa opalina oscura en diatomitas. En el borde exterior de la capa (flechas) se diferencia una zona más compacta de diatomitas carbonatadas. Los tonos claros del interior de la capa también son zonas carbonatadas (flecha); E. Corte de un nódulo opalino y su borde exterior. Zona 1, diatomita calcificada. Zonas 2 y 3 , mezclas de ópalo y calcita provenientes de la calcificación de diatomeas u ópalo. Zona 4, ópalo; F. Microfotografía de la zona 1 en Figura E. La mayoría de las diatomeas están total o parcialmente calcificadas (el tono rojo es por la tinción de la calcita con alizarina). Microscopia óptica y nícoles paralelos.

\section{Metodología}

Se tomaron muestras de los diferentes tramos de diatomeas calcificadas y de las diatomitas y ópalos asociados. El estudio mineralógico se llevó acabo con Difracción de Rayos X (DRX), mediante diagramas de polvo total, con un Equipo PHILIPS modelo PW1710, equipado con monocromador de grafito. La mineralogía puntual sobre diatomeas calcificadas específicas, se realizó con micro-Raman, utilizando un microscopio confocal con espectrómetro Thermo Fisher. El estudio petrológico fue realizado por microscopía óptica convencional y electrónica de barrido (MEB). En MEB, se trabajó sobre fragmentos de muestras metalizadas con oro, con un equipo FEI Inspect, llevándose a cabo a la vez análisis químicos puntuales por energía dispersiva (EDS), con un equipo OXFORD 
Inca. Todos los instrumentos utilizados pertenecen al Museo Nacional de Ciencias Naturales (CSIC) de Madrid.

\section{Mineralogía y Petrología}

Los depósitos de partida en ambas secciones son diatomitas, pero las características composicionales y texturales de las secciones estudiadas son muy distintas.

\section{Sección de Tresjuncos (Fig. 1)}

Las calizas/dolomías resultantes de la carbonatación de las diatomitas presentan una fina laminación que refleja la estructura de la primitiva diatomita, preservándose frecuentemente intercalaciones submilimétricas de diatomitas no reemplazadas.

En DRX se detecta que las diatomitas están constituidas principalmente por ópalo A (sílice amorfa proveniente de los microfósiles silíceos), con indicios ocasionales de calcita o dolomita. Las rocas carbonáticas están constituidas por calcita o dolomita (90-95\%), detectándose minoritariamente paligorskita, cuarzo y en algún caso yeso. En los análisis de DRX aparece calcita o dolomita, pero no coexisten ambas. En las calizas la reflexión principal de la calcita se marca a $3.04 \AA$ indicando que no existe $\mathrm{Mg}$ en la red. En micro-Raman, las valvas calcificadas además de calcita o dolomita, presentan indicios de carbono orgánico que queda reflejado por la banda $\mathrm{G}$ alrededor de $1.595 \mathrm{~cm}^{-1}$ (Brolly et al., 2016) (Figs. 3 A y B).

En el MEB se observa que las diatomitas no presentan cemento, existiendo una matriz detrítica formada por los mismos fragmentos de las frústulas de diatomeas (Fig. 4A). Además de diatomeas, ocasionalmente se encuentran espículas silíceas.

Las capas de calizas formadas a partir de diatomitas muestran frecuentemente valvas carbonatadas o sus moldes (Figs. 4B y C). Grandes cristales de calcita se pueden ver cementando huecos (Figs. 4B y D). Es común que películas de arcillas fibrosas, cuyas composiciones coinciden con paligorskita o sepiolita alumínica, recubran tanto las diatomeas calcificadas como los cristales euhedrales de calcita que constituyen cementos (Fig.4D).

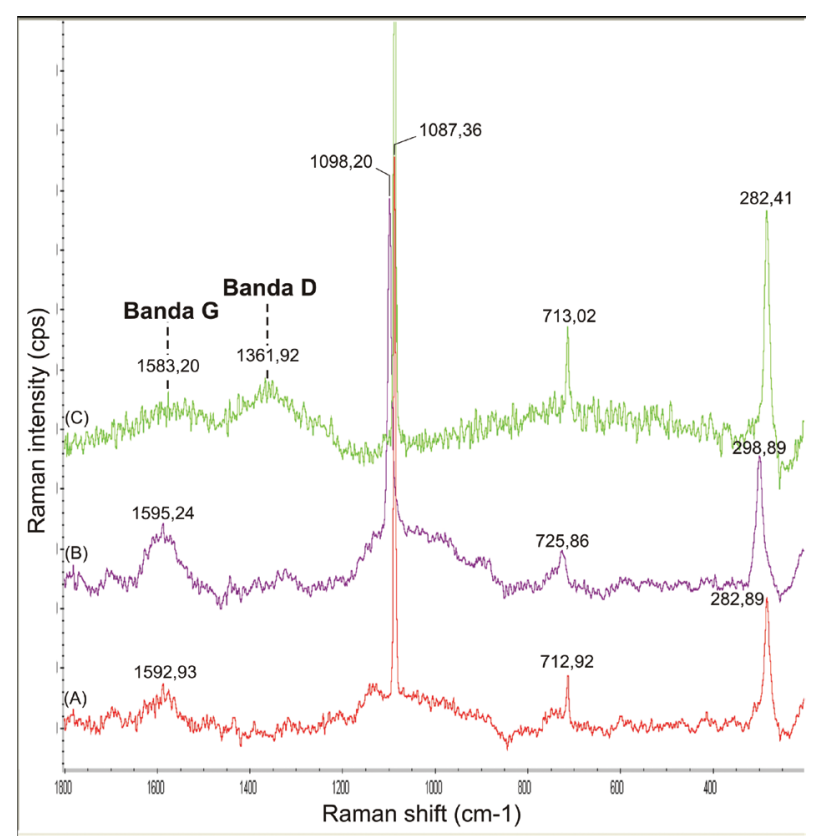

Figure 3.-Espectros micro-Raman realizados sobre diatomeas reemplazadas por calcita o dolomita y bandas $G$ y $D$ de la materia orgánica. A (valva calcitizada) y B (valva dolomitizada), (Tresjuncos). C (valva calcitizada), Níjar.

Las capas de dolomías generadas a partir de las diatomitas presentan cementos/matriz de cristales romboédricos de dolomita con tamaño inferior a $4 \mu \mathrm{m}$ dejando ver claramente las diatomeas remplazadas (Fig. 4E). Incluyen localmente arcillas fibrosas cuya composición encaja con la paligorskita o sepiolita alumínica (Fig. 4E). Las valvas de las diatomeas reemplazadas presentan sus areolas (perforaciones en la frústula) cementadas por cristales de dolomita que adquieren el tamaño de los huecos (Figs. 4 E, F y G). Las partes no ornamentadas de la valva (partes hialinas) presentan aspecto compacto, detectándose en EDS una coexistencia de $\mathrm{Ca}, \mathrm{Mg}$ y Si (Fig. 4G); esto indicaría que todavía quedan restos opalinos dentro de ellas, ya que no aparecen arcillas fibrosas. En ciertas zonas, se observan secciones hexagonales o idiomorfas de dolomita (Figs. 4F y G) que pueden incluir varias areolas cementadas por dolomita.

\section{Sección de Níjar (Fig. 1)}

La sección estudiada está formada por tramos diatomíticos con capas lenticulares y nódulos opalinos, $\mathrm{y}$ tramos de biocalcilutitas $\mathrm{y}$ 
M.A. Bustillo

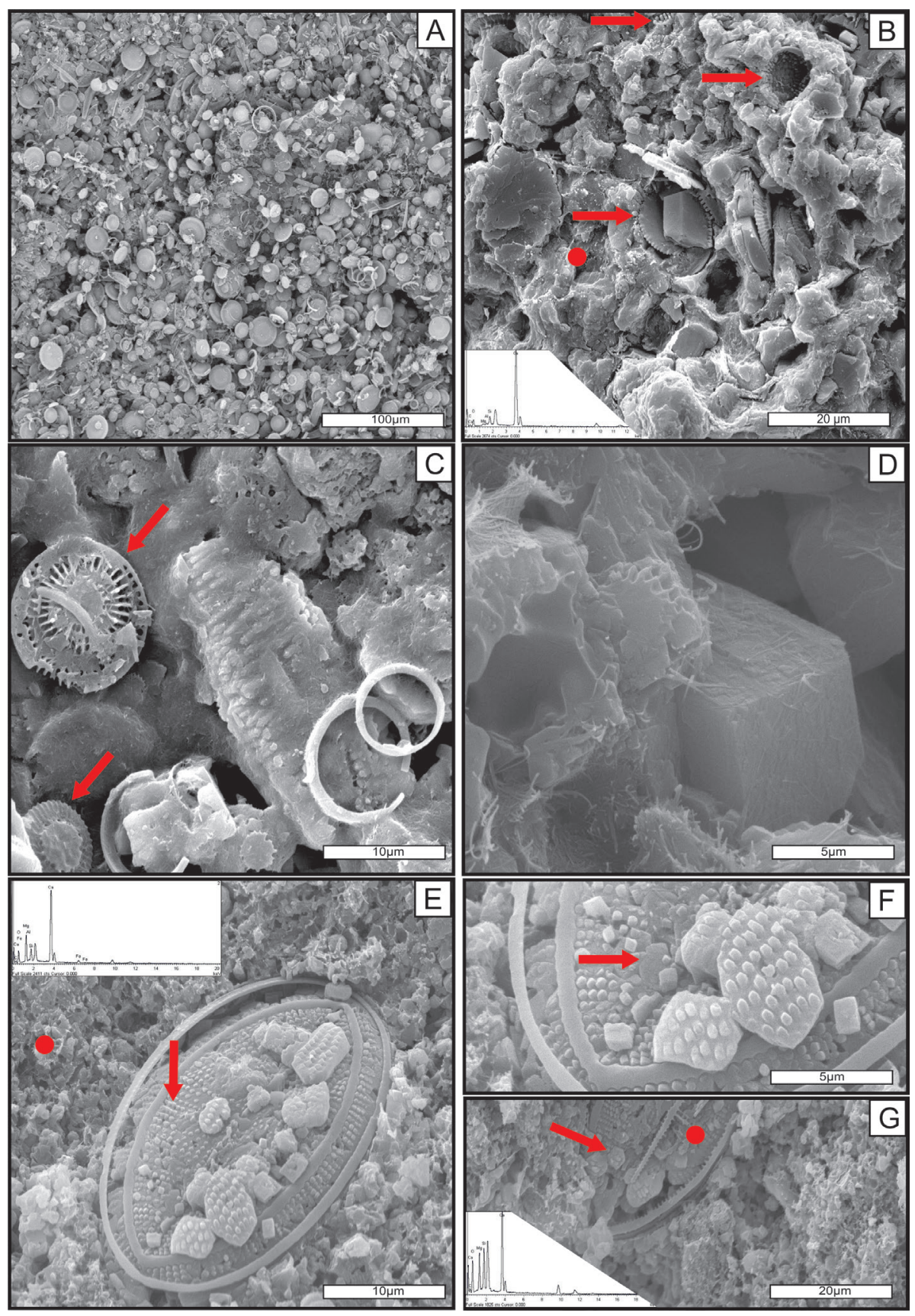

Figure 4.-Diferentes aspectos en MEB y EDS de diatomitas y calizas o dolomías diagenéticas. Sección de Tresjuncos. A. Diatomita formada mayoritariamente por Cyclotella iris Brun and Heribaud, 1893; B. Huellas de diatomeas calcificadas y moldes esféricos (flechas) en caliza diagenética. Los análisis de EDS (punto rojo) registran además de calcita la presencia de paligorskita o sepiolita alumínica (Pal-Sep); C. Posibles Cyclotellas iris calcificadas, y películas de Pal-Sep recubriéndolas; D. Películas de Pal-Sep recubren tanto las diatomeas como los cristales euhedrales de calcita que cementan la roca; E. Visión exterior de una valva de Cocconeis placentula Ehrenb. 1838, dolomitizada incluida en dolomicrita con Pal-Sep (EDS en punto rojo). Las areolas de la valva están rellenas por cristales pequeños de dolomita que resaltan en la valva; F. Detalle de Figura E, donde se observan cristales de dolomita de sección hexagonal, en la zona hialina de la valva (flecha); G. Visión interior de una valva de Cocconeis placentula, con cristales de dolomita de sección hexagonal (flecha). Puntualmente se detecta todavía Si con EDS en la valva (punto rojo). 
biocalcarenitas (Fig.1D). En los tramos diatomíticos también hay algunas laminas /capas milimétricos/ centimétricas de biocalcilutitas y biocalcarenitas. Son packestones o wackstones de microforaminíferos, nanoplacton calcáreo... etc., y en ocasiones con fragmentos de equinodermos, briozoos y moluscos. Aparecen en estas láminas/capas procesos de silicificación poco intensos quedando patentes en la silicificación de la matriz carbonática y en el relleno por ópalo de ciertos microfósiles carbonáticos.

Las diatomitas pueden presentar además de diatomeas, pequeñas proporciones de otros microfósiles silíceos (esponjas silíceas, radiolarios, silicoflagelados...etc.). Los microfósiles calcáreos siempre están presentes aunque sea en pequeña proporción. En DRX, además de ópalo A y calcita se han encontrado indicios de yeso, cuarzo, feldespatos y micas.

Las zonas más compactas que rodean ciertos capas lenticulares y nódulos opalinos (Figs. 2D y E) son muy complejas. Los estudios en DRX revelan composiciones de calcita en su parte más exterior (zona 1, Fig. 2E), mezclas de ópalo CT y calcita con indicios $(5-10 \%)$ de yeso y micas en las bandas intermedias (zonas 2 y 3, Fig. 2E), y ópalo CT en el interior del nódulo (zona 4, Fig. 2E). En el borde de carbonatación (zona 1) solo aparece calcita con su reflexión principal a $3.03 \AA$., pudiendo indicar un $2 \%$ de moles de $\mathrm{MgCO} 3$ en la red. (Goldsmith et al., 1961).

En lámina delgada, y con tinción con alizarina, se observa que la zona 1 está constituida por diatomeas total o parcialmente carbonatadas (Fig. 2F). Las zonas 2 y 3 son diatomitas carbonatadas silicificadas por ópalo y ópalos carbonatados. La zona 4 es completamente opalina, observándose la fina laminación de las diatomitas originales, y la presencia de algún microfósil calcáreo. Esta estructura compleja de bandas alrededor de los nódulos/capas indica reemplazos reversibles de calcita y ópalo. Puntualmente en todas las bandas aparecen indicios de óxidos de Fe.

Los análisis de micro-Raman realizados sobre las valvas de diatomeas calcificadas de la zona 1 muestran que además de calcita aparecen indicios de carbono orgánico que se detecta por la presencia de la banda $\mathrm{G}$ (alrededor de $1.583 \mathrm{~cm}^{-1}$ ) y posiblemente la banda D (alrededor de $1.361 \mathrm{~cm}^{-1}$ ). La intensidad de la banda D (mayor que la de la banda G) (Fig. 3C) puede ser debida a la influencia de hematites, que presentan una banda en una frecuencia próxima (Brolly et al., 2016).

En las diatomitas las valvas de las diatomeas normalmente muestran signos menores de disolución (Fig. 5A). Solo existen microporos muy localizados (Fig. 5B) y la sílice resultante de esta disolución puede ser la fuente de las pequeñas lepiesferas de ópalo CT que se forman sobre ellas mismas. En otras ocasiones se observa recubrimientos importantes de sílice sobre las valvas (Fig. 5C) indicando soluciones intersticiales ricas en sílice, y mayor precipitación de ópalo CT.

En las zonas carbonatadas, las valvas manifiestan características diferentes. Una veces presentan una estructura de nido de abeja hexagonal con la areola (poro) centrada en el interior del hexágono y apareciendo $\mathrm{Ca}$ en EDS (Fig. 5D). Estas formas hexagonales corresponden a las cámaras hexagonales que se encuentran debajo de las areolas y que en las valvas silíceas no son tan evidentes (Fig. 5A). Se interpreta, entonces, que la morfología hexagonal y la presencia de $\mathrm{Ca}$ en la composición de las valvas indican un reemplazado del ópalo por calcita a escala micrométrica. Otras veces se observan cristales de calcita euhedrales (Figs. 5E y F), cementando las areolas. Ambos procesos el reemplazo y la cementación, pueden coexistir en las valvas calcificadas, como lo demuestra la existencia de $\mathrm{Si}$ y $\mathrm{Ca}$ en las partes hialinas que aparecen entre las areolas cementadas (Fig. 5F).

En las especies con frústulas complejas el pseudomorfismo queda patente porque se refleja bien cada una de sus complejidades. Valvas silíceas con diferentes tamaños de areolas y cíngulos perforados (Fig. 6A), después de calcificarse (Fig. 6B) muestran que el cíngulo con su fina perforación se ha reemplazado por grandes cristales de calcita con sección hexagonal y que los poros se han cementado por diminutos cristales de calcita (Fig. 6C). La composición química de los cristales de calcita, obtenida por EDS, revela que no incluye Mg (Fig. 6B). Algunas valvas calcificadas pueden posteriormente sufrir procesos de silicificación puntuales que se manifiestan por la aparición de lepiesferas de ópalo sobre la valva calcificada (Fig. 6D). Localmente se observa, en la roca diatomítica carbonatada, algún cristal de calcita como cemento entre las valvas calcificadas. 

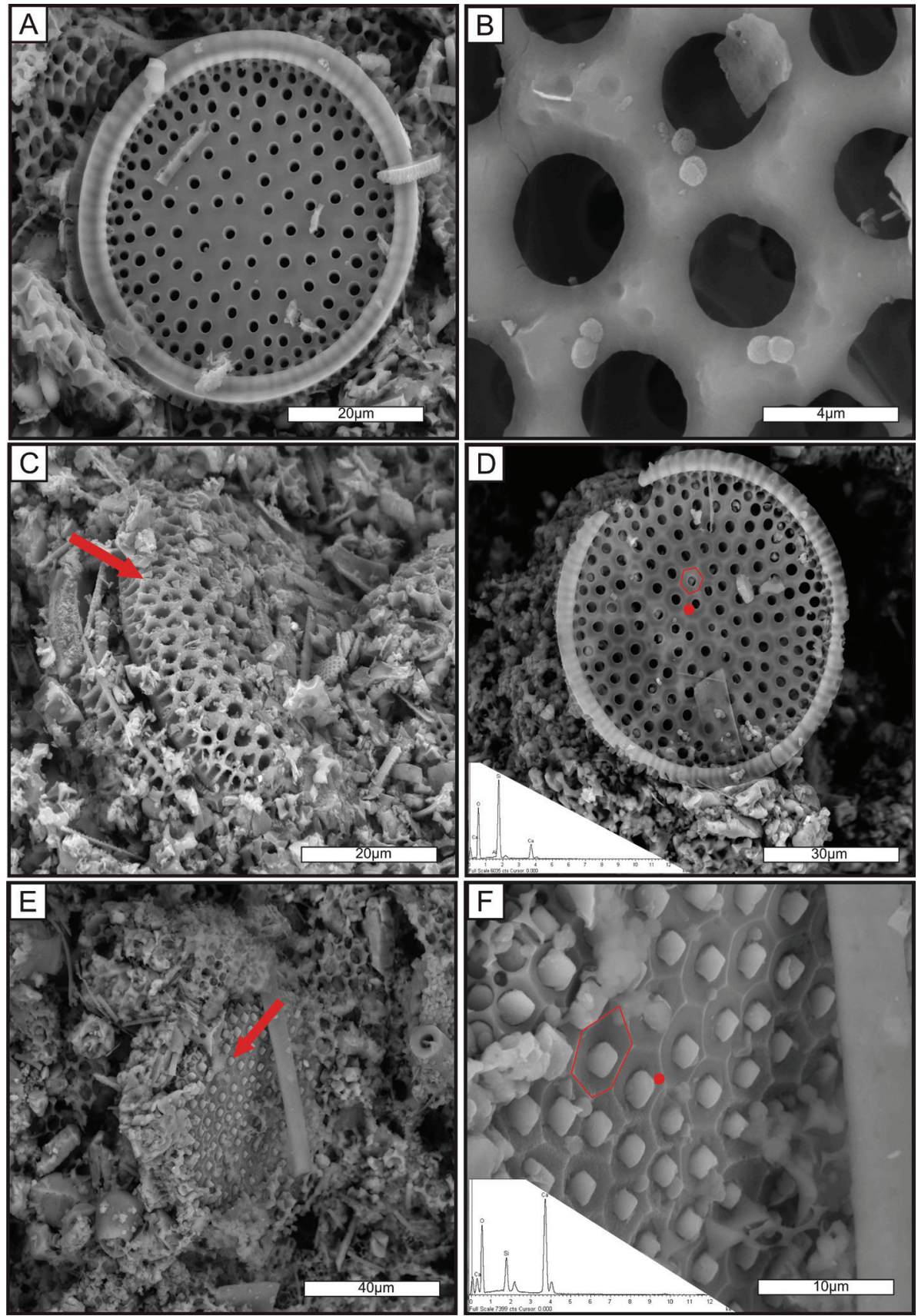

Figure 5.-Diferentes aspectos en MEB de diatomeas y su carbonatación. Sección de Níjar. A. Visión interna de una valva opalina de Coscinodiscus aff. marginatus Ehrenberg 1844; B. Detalle de una valva con pequeños microporos de disolución y nueva reprecipitación de sílice como pequeñas lepiesferas de ópalo CT; C. Frústula de diatomea ( posible Coscinodiscus ), observándose un recrecimiento por ópalo; D. Valva de Coscinodiscus aff. Marginatus, observándose la estructura del panel de abeja correspondiente a las cámaras hexagonales de las areolas (contorno de una cámara dibujado en rojo). Presenta cierta proporción de Ca indicando reemplazo parcial (análisis de EDS en punto rojo); E. Valva de diatomea céntrica calcificada. Las areolas están cementados por cristales de calcita (flecha); F. Detalle de la foto anterior mostrando la estructura de cámaras hexagonales (contorno de una de ellas dibujado en rojo) entre las areolas ya cementadas por calcita. La zona hexagonal todavía conserva algo de la sílice de la valva (análisis de EDS en punto rojo). 

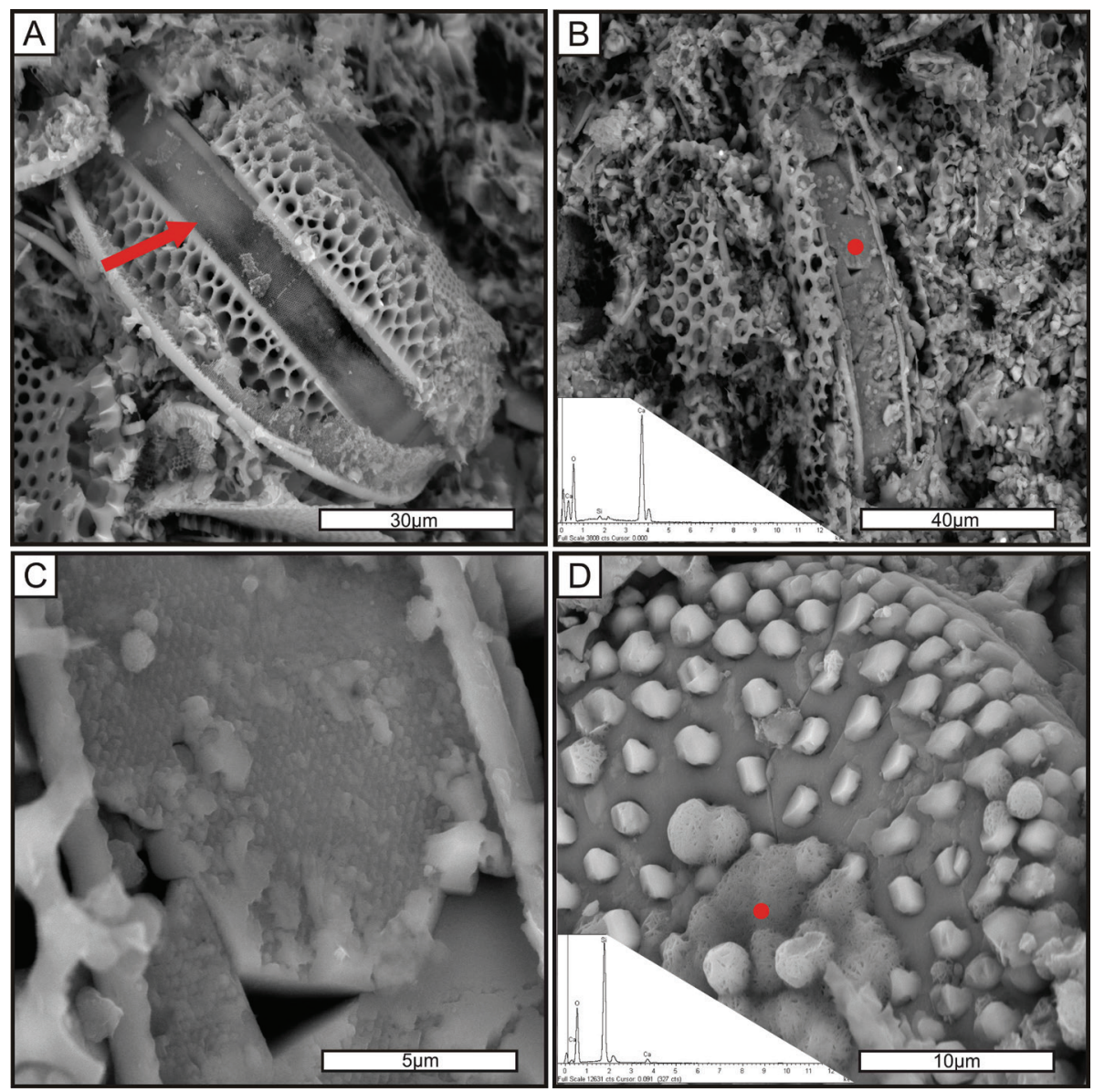

Figure 6.-Diferentes aspectos en MEB de diatomeas y su carbonatación. Sección de Nijar. A. Frústula de Coscinodiscus sp., con las dos valvas unidas por el cíngulo perforado; B. Frústula carbonatada de Coscinodiscus sp. con las dos valvas y el cíngulo. Se observa grandes cristales de calcita (ver el análisis en EDS, punto rojo) en el cíngulo; C. Detalle de la foto anterior donde se ve las secciones hexagonales de calcita que incluyen otros minúsculos $(\leq 0.5 \mu \mathrm{m})$ también de calcita, reflejando la cementación de la perforación del cíngulo; D. Visión exterior de una valva de diatomea calcificada y posteriormente silicificada por lepiesferas de ópalo CT. Véase la riqueza en Si del espectro de EDS en las lepiesferas (punto rojo).

\section{Discusión}

\section{Los procesos de reemplazo}

Los datos morfológicos y texturales obtenidos en la observación de las valvas de diatomeas carbonatadas revelan que, tanto en la sucesión sedimentaria continental como en la marina, la trasformación de las diatomitas a calizas o dolomías se da por procesos de reemplazo y cementación.

En las valvas reemplazadas por calcita o dolomita se conserva la totalidad del volumen y una gran parte de las características texturales y estructurales, por lo que se trata de un reemplazo pseudomórfico. Reacciones de reemplazos pseudomórficos se dan en la Naturaleza entre diferentes minerales, tanto en condiciones de alta temperatura y presión como en condiciones ambientales. En el sistema mineral de la sílice-carbonatos, estos reemplazos se realizan por procesos de disolución-precipitación en fluidos acuosos. Se trata de reacciones de disolución y precipitación acopladas en el espacio y en el tiempo (Brugger et al., 2010), donde la fase inicial que se disuelve está incluida dentro del mismo fluido en el que se produce la precipitación de la nueva fase, y todo ello en el mismo frente de reacción.

Los procesos de silicificación de carbonatos son ampliamente conocidos y descritos en la literatura científica (Bustillo, 2010; Liesegang et al., 2017), sin embargo, los procesos inversos de carbonatación 
de depósitos silíceos son mucho menos frecuentes y poco estudiados. Harker (1971) experimenta en el laboratorio con diferentes valvas de diatomeas, sometiéndolas a diferentes temperaturas (desde ambientales hasta $600^{\circ} \mathrm{C}$ ) en presencia de óxido de calcio y $\mathrm{CO}_{2}$. Preferentemente, en la especie Coscinodiscus, consigue el reemplazo de la sílice por calcita, mostrando que se conserva la estructura de las valvas y sus areolas. Este autor, identifica con microscopia óptica cristales aislados de calcita, pero también, mediante DRX, fases poco cristalinas de silicatos cálcicos hidratados y tobermorita $\left(\mathrm{Ca}_{5} \mathrm{Si}_{6} \mathrm{O}_{16}(\mathrm{OH})_{2} \cdot \mathrm{nH}_{2} \mathrm{O}\right)$

Los procesos de reemplazo y cementación reconocidos mediante SEM en las valvas estudiadas concuerdan con los datos morfológicos obtenidos por Harker (1971), ya que además de la reproducción de todas las características estructurales de las valvas, en ocasiones, se pueden ver grandes cristales de calcita (Figs. 6 B y C) o dolomita (Figs. 4 E, F y G). Los estudios de EDS reflejan pequeñas proporciones de $\mathrm{Si}$ asociadas a los cristales de calcita o dolomita (Figs. 4G y 6B) indicando que permanecen relictos de las microesferas de ópalo amorfo de las valvas. No se considera la presencia de silicatos cálcicos porque no aparecen ni en DRX ni en micro-Raman.

La formación directa de cristales idiomorfos de calcita sobre ópalo se ha explicado por su semejanza con la formación de calcita idiomorfa en los ensayos de crecimiento de cristales en geles de sílice, ya que la estructura del ópalo amorfo es parecida a los geles que sirven de base al crecimiento (Harker 1971). Este proceso también ha sido descrito en ópalos inorgánicos (Bustillo \& La Iglesia, 1978; Bustillo \& Ruiz Ortiz 1987), donde además de calcita se puede formar dolomita. Actualmente se podría considerar las hipótesis de crecimiento de cristales calcita a partir de la agregación de nanopartículas o complejos amorfos de varios iones (De Yoreo et al., 2015). La acumulación de estas partículas/complejos de un carbonato amorfo precursor subsecuentemente se transformaría en un cristal de calcita o dolomita. La estructura cristalina del ópalo de las frústulas (numerosas microesferas de sílice amorfa de tamaño nanométrico/micrométrico) facilita, por una parte, la disolución de la sílice, y por otra, la precipitación de múltiples partículas nanopartículas/complejos multi-iónicos de carbonato. Las nanopartículas o complejos carbonáticos habrían seguido las orientaciones de las microesferas de sílice amorfa que conforman la valva, conservándose así todas sus características morfológicas.

Las curvas de solubilidad de la sílice y la calcita en relación al $\mathrm{pH}$, y a temperatura ambiente, se han utilizado por numerosos autores para explicar los procesos de reemplazo en el sistema calcita-sílice (Bustillo, 2010). Cuando el pH excede de 9, la solubilidad de la sílice aumenta drásticamente; si el pH cae a un valor más bajo, se precipita. Una solubilidad inversa tiene la calcita en estos márgenes de $\mathrm{pH}$ y cuando la sílice se disuelve, la calcita precipita. De esta manera, cuando hay pequeñas variaciones de $\mathrm{pH}$ en un entorno alrededor de 9, los procesos pueden ser reversibles, como se observa en la estructura compleja que aparece en el exterior de los nódulos de la sección de Níjar. Por otra parte, la presencia de sulfatos, puede facilitar la disolución de la sílice, (Bai et al., 2009) no siendo necesarios pH tal altos como 9.

\section{Ambientes y condiciones diagenéticas}

Las condiciones en las que se produce la carbonatación de las diatomeas son diferentes si consideramos que este proceso sucede tanto en la diagénesis temprana de ambiente continental (sección de Cerro Molino, Tresjuncos, Bustillo et al., 2017b), como en la diagénesis marina por enterramiento (sección de Níjar)

\section{Sección de Tresjuncos}

En esta sección continental no existen procesos de formación de ópalo que podrían ser desencadenantes de una carbonatación, como sucede en la sección de Níjar. Sin embargo, las características comunes que comparte con la sección de Níjar son: 1) Asociación de las diatomitas, en lateral y en vertical, con depósitos carbonaticos (wackestones/packstones de microorganismos calcáreos, Bustillo et al., 2017b), 2) existencia de materia orgánica, detectada por Micro-Raman, en las diatomeas calcificadas y 3 ) presencia de yeso intersticial en pequeñas cantidades. La fuente de $\mathrm{C}$ y Ca podría estar en las calizas contiguas que por disolución/recristalización durante la diagénesis pueden aportar el $\mathrm{C}$ y $\mathrm{Ca}$ a las 
capas diatomíticas próximas. Sin embargo también se podría considerar la influencia de una sulfatoreducción, que es una de las causas expuestas por Hein et al. (1979) para que se genere una fuente de C y se faciliten los procesos de reemplazo de valvas de diatomeas. Los depósitos diatomíticos lacustres que muestran calcita o dolomita por reemplazo, incluyen numerosos organismos fosilizados (plantas, artrópodos, anfibios...etc.), yeso en pequeña proporción y tapices microbianos (Bustillo et al., 2017b). La existencia de organismos degradándose existiendo putrefacción de su materia orgánica, la presencia de yeso, y la porosidad y permeabilidad de las diatomitas facilitarían los procesos de reemplazado de las valvas. La presencia de dolomita en los insectos fosilizados incluidos en las diatomitas se ha explicado por procesos de sulfato reducción (Bustillo, 2018). En este ambiente, el $\mathrm{CO}_{2}$ pudo derivarse de la degradación bacteriana de la materia orgánica que condujo a la formación de iones bicarbonato que al reaccionar con los iones presentes en el fluido intersticial pueden producir carbonatos autigénicos. En este contexto, los cambios de $\mathrm{pH}$ y salinidad pudieron ser cruciales para los procesos de reemplazo sílice-carbonatos, que dependiendo de la presencia o no de $\mathrm{Mg}$, generaron calcita o dolomita a partir de las valvas.

Los procesos de fosilización en calcita (anfibios) o dolomita (insectos) que se producen en las diatomitas se han considerado diagenéticos tempranos porque estarían facilitados por la degradación de la materia orgánica (Bustillo et al., 2017b; Bustillo 2018). Es factible que las carbonataciones de las valvas de diatomeas sean coetáneas con estas fosilizaciones y por lo tanto también diagenéticas tempranas.

\section{Sección de Nijar}

En la sección marina, la fuente de C y Ca estaría principalmente en los microfósiles calcáreos incluidos en las diatomitas. Como consecuencia de la formación de las capas lenticulares y nódulos opalinos por procesos de envejecimiento de las diatomitas, se producen reemplazos de los microorganismos calcáreos por sílice, quedando libre $\mathrm{CO}_{2}$ y Ca. El carbono orgánico detectado en micro-Raman sobre las diatomeas calcificadas podría ser un residuo de la materia orgánica existente en las diatomeas, y que también pudo haber contribuido a la fuente de carbono. A su vez hay que considerar que las diatomitas están intercaladas entre capas de biocalcarenitas/biocalcilutitas que por disolución/recristalización durante la diagénesis pueden aportar el $\mathrm{C}$ y $\mathrm{Ca}$ a las capas diatomíticas contiguas.

La composición química detectada en EDS revela que los cristales autigénicos de calcita no incluyen ningún elemento, ni siquiera $\mathrm{Mg}$. La fuente de los carbonatos se encontraría en el fluido intersticial. Una subida en el pH en el fluido intersticial, después de la disolución de los microfósiles calcáreos, favorecería la disolución de las diatomeas y la precipitación de los carbonatos (Hein et al., 1979). La presencia de pequeñas cantidades de yeso, tanto en las diatomitas como en el borde de los nódulos opalinos, podría indicar la presencia de sulfatos en el fluido intersticial, que a su vez influirían en el proceso de reemplazo por facilitar la disolución del ópalo. De acuerdo con Bai et al.(2009) el grado de disolución de la sílice amorfa es acelerada por la presencia de sulfatos debido a la adsorción de iones $\mathrm{SO}_{2}^{-4}$ sobre la superficie de la sílice, ya que los enlaces $\mathrm{Si}-\mathrm{O}$ del ópalo se debilitan.

Determinar el momento diagenético de los procesos de calcificación es problemático. Calcificaciones de diatomeas marinas y otras carbonataciones alrededor de nódulos de sílex han sido también señaladas por otros autores (Hein et al., 1990) sin abordar su estudio. Procesos similares podrían ser los de carbonatación de espículas silíceas y radiolarios incluidos en calizas de plataformas marinas y en depósitos marinos profundos de diferentes edades (Meyers, 1977; Maliva \& Siever, 1989; Bustillo \& Ruiz Ortiz, 1987; Bustillo et al., 2017a). En estos casos, se considera que las carbonataciones son procesos diagenéticos tempranos, pero esto no se puede extrapolar al caso estudiado. Hay que pensar que la calcificación de un organismo silíceo, cuando este está incluido en un depósito carbonático, tiene que ser un proceso diagenético temprano porque las frústulas de sílice amorfa (ópalo A) son muy inestables en estas circunstancias y si no se calcifican rápidamente se disuelven (Bustillo, 2010). No sucede lo mismo cuando se trata de depósitos silíceos (diatomitas, radiolaritas... etc.) porque en ellos al disolverse las especies más débiles, enriquecen en sílice los 
fluidos intersticiales y el resto de especies quedan protegidas pudiendo permanecer tal cual a grandes profundidades de enterramiento y luego ser calcificadas. Por lo tanto, es difícil determinar el momento diagenético de los procesos de calcificación de la sección estudiada, más allá de que son posteriores a la formación de las capas lenticulares y nódulos opalinos, que también aparecen puntualmente calcificados.

\section{Conclusiones}

Los procesos de carbonatación de diatomeas son procesos pseudomórficos que se llevan a cabo mediante el reemplazo directo del ópalo A de la frústula por calcita o dolomita. Coetáneamente al reemplazo se producen cementaciones de las areolas y otras perforaciones intrínsecas de cada frústula de acuerdo con su especie. La perfecta reproducción de las valvas indica reacciones de disoluciónprecipitación acopladas en el espacio y tiempo. La misma organización de las microesferas de ópalo A dentro de las valvas podría condicionar la organización de complejos multiionícos/ nanopartículas de carbonatos amorfos que posteriormente recristalizarían a calcita o dolomita. Estas características hacen que el proceso de carbonatación de diatomeas sea un ejemplo de secuestro natural de $\mathrm{CO}_{2}$ sin cambio de volumen, siendo de gran interés para considerar el estudio de las diatomitas como almacén subterráneo de $\mathrm{CO}_{2}$.

Las condiciones y causas por las que se produce la carbonatación de las diatomeas son diferentes durante la diagénesis temprana en ambiente continental poco profundo (Tresjuncos) y la diagénesis por enterramiento de la serie marina (Níjar). En la sección continental, el proceso de carbonatación de las valvas se considera favorecido por la materia orgánica que tuvieron las diatomitas lacustres, ya que incluyen numerosos restos de organismos. En este ambiente, los procesos de sulfato-reducción durante la degradación de la materia orgánica de los diferentes organismos, generarían $\mathrm{CO}_{2}$, que junto con cambios de $\mathrm{pH}$ y salinidad facilitaron los reemplazos de las valvas. Se considera posible que las carbonataciones de las valvas sean coetáneas a la formación de calcita y dolomita durante las fosilizaciones de los organismos, y por lo tanto, diagenéticas tempranas. En la sección marina el proceso desencadenante del reemplazo de las valvas de diatomeas es la formación de capas lenticulares y nódulos opalinos en las diatomitas porque estas incluyen intercalaciones de biocalcarenitas/ biocalcilutitas. El reemplazo de los microorganismos calcáreos por sílice en las capas/nódulos opalinos genera $\mathrm{CO}_{2}$ y $\mathrm{Ca}$ en el fluido intersticial. Pequeñas variaciones de $\mathrm{pH}$ en un entorno alrededor de 9, y la presencia de sulfatos facilitarían el reemplazo de las valvas, durante la diagénesis por enterramiento.

\section{AGRADECIMIENTOS}

Este trabajo ha sido financiado por el proyecto de investigación CGL2014-54818-P (Ministerio de Economía, Industria y Competitividad / FEDER, Unión Europea). La autora agradece la ayuda recibida de $\mathrm{M}^{\mathrm{a}}$ José Lopez Garcia en la identificación de diatomeas. Igualmente expresa su reconocimiento al equipo técnico de los laboratorios del Museo Nacional de Ciencias Naturales de Madrid y particularmente a Manuel Castillejo (preparación muestras), Rafa González (DRX), Laura Tormo, Marta Furió, y Pedro Valverde (MEB, EDS, micro-Raman) y a Alvaro Moreno (edición gráfica de este trabajo). Asimismo agradece a los revisores científicos Ildefonso Armenteros y Esther Sanz los comentarios y sugerencias que han mejorado el manuscrito original.

\section{Referencias}

Bai, S.; Urabe, S.; Okaue, Y. \& Yokoyama, T. (2009). Acceleration effect of sulfate ion on the dissolution of amorphous silica. Journal of colloid and interface Science, 331: 551-554. https://doi.org/10.1016/j. jcis.2008.11.076

Bellanca, A.; Calvo, J. P.; Censi, P.; Elizaga, E. \& Neri, R. (1989). Evolution of lacustrine diatomite carbonate cycles of Miocene age, southeastern Spain; petrology and isotope geochemistry. Journal of Sedimentary Research, 59: 45-52. https://doi.org/10.1306/ 212F8F12-2B24-11D7-8648000102C1865D

Bickle, M.; Kampman, N.; Chapman, H.; Ballentine, C.; Dubacq, B.; Galy, A. \& Zhou, Z. (2017). Rapid reactions between $\mathrm{CO} 2$, brine and silicate minerals during geological carbon storage: Modelling based on a field $\mathrm{CO} 2$ injection experiment. Chemical Geology, 468: 17-31. https://doi.org/10.1016/j. chemgeo.2017.07.031

Brolly, C.; Parnell, J. \& Bowden, S. (2016). Raman spectroscopy: Caution when interpreting organic carbon from oxidising environments. Planetary and space Science, 121: 53-59. https://doi.org/10.1016/j. pss.2015.12.008 
Brugger, J.; McFadden, A.; Lenehan, C. E.; Etschmann, B.; Xia, F.; Zhao, J. \& Pring, A. (2010). A novel route for the synthesis of mesoporous and low-thermal stability materials by coupled dissolution-reprecipitation reactions: mimicking hydrothermal mineral formation. CHIMIA International Journal for Chemistry, 64: 693-698. https://doi.org/10.2533/chimia.2010.693

Bustillo, M.A. (2010). Silicification of continental carbonates. En: Carbonates in Continental Setting. Processes, Facies and Applications (Alonso-Zarza, A.M. \& Tanner, L.H.,Eds.). Elsevier, Amsterdam. Developments in Sedimentology 62: 153-174. https://doi. org/10.1016/S0070-4571(09)06203-7

Bustillo, M. A. (2018). Formación de biodolomita durante un proceso de fosilización de insectos en diatomitas miocenas (Konservat-Lagerstätte Tresjuncos, Cuenca, España). Geogaceta, 64: 127-130.

Bustillo, M. \& La Iglesia, A. (1978). Procesos de formación y diagénesis en las rocas silíceas de Sagunto. Estudios Geológicos, 34: 167-174.

Bustillo, M. A. \& Ruiz-Ortiz, P. A. (1987). Chert occurrences in carbonate turbidites: examples from the Upper Jurassic of the Betic Mountains (southern Spain). Sedimentology, 34: 611-662. https://doi. org/10.1111/j.1365-3091.1987.tb00790.x

Bustillo, M.; Elorza, J. \& Díez-Canseco, D. (2017a). Silicificaciones selectivas en Thalassinoides y otras estructuras biogénicas asociadas a calizas de plataforma marina y hardground (Albiense inferior, Sonabia, Cantabria). Estudios Geológicos 73(1): e064. https://doi.org/10.3989/egeol.42668.435

Bustillo, M.A.; Díaz-Molina, M.; López-García, M.J.; Delclòs, X.; Peláez-Campomanes, P.; Peñalver, E.; Rodríguez-Talavera, R. \& Sanchiz, B. (2017b). Geology and paleontology of Tresjuncos (Cuenca, Spain), a new diatomaceous deposit with Konservat-Lagerstätte characteristics from the European late Miocene. Journal of Iberian Geology, 43: 395411. https://doi.org/10.1007/s41513-017-0032-4

Calvo, J.P.; Pozo, M. \& Servant-Vildary, S. (1988). Lacustrine diatomite deposits in the Madrid Basin (Central Spain). Geogaceta, 4: 14-17.

De Yoreo, J.J.; Gilbert, P.U.; Sommerdijk, N.A.; Penn, R.L.; Whitelam, S.; Joester D.; Zhang D.H.; Rimer J.D.; Navrotsky,A.; Banfield J.F.; Wallace, A.F.; Michel M.; Meldrum F.C.; Cölfen H. \& Dove P.M. (2015). Crystallization by particle attachment in synthetic, biogenic, and geologic environments. Science, 349: aaa6760. https://doi.org/10.1126/science.aaa6760

Díaz-Molina, M. \& Tortosa, A. (1996). Wet fluvial fans of the Loranca Basin, Upper Oligocene-Lower Miocene, Central Spain. In: Tertiary basins of Spain: the stratigraphic record of crustal kinematics (Friend P. \& Dabrio C., Eds.), Cambridge University Press, Cambridge, UK, 300-307. https://doi.org/10.1017/ CBO9780511524851.042

Flaathen, T. K.; Oelkers, E. H.; Gislason, S. R. \& Aagaard, P. (2011). The effect of dissolved sulphate on calcite precipitation kinetics and consequences for subsurface CO2 storage. Energy Procedia, 4: 5037-5043. https://doi.org/10.1016/j.egypro.2011.02.476

Gómez, J. J.; Díaz-Molina, M. \& Lendínez, A. (1996). Tectono-sedimentary analysis of the Loranca Basin (Upper Oligocene-Miocene, central Spain). A non-sequenced foreland basin. In: Tertiary basins of Spain: The stratigraphic record of crustal kinematics (Friend P. \& Dabrio C. Eds.), Cambridge University Press, Cambridge, UK, 277-286. https://doi. org/10.1017/CBO9780511524851.040

Harker, R. I. (1971). Synthetic calcareous pseudomorphs formed from siliceous microstructures. Science, 173(3993): 235-237. https://doi.org/10.1126/ science.173.3993.235

Hein, J. R.; O’Neil, J. R. \& Jones, M. G. (1979). Origin of authigenic carbonates in sediment from the deep Bering Sea. Sedimentology, 26: 681-705 https://doi. org/10.1111/j.1365-3091.1979.tb00937.x

Hein, J. R.; Yeh, H. W. \& Barron, J. A. (1990). Eocene diatom chert from Adak Island, Alaska. Journal of Sedimentary Research, 60: 250-257. https://doi.org/10.1306/212F9165-2B24-11D78648000102C1865D

Hernáiz, P.P. \& Cabra, P. (1998). Mapa Geológico de España a Escala 1:50.000, hoja n ${ }^{\circ} 661$ (Villarejo de Fuentes) y memoria, ITGE, Madrid.

Liesegang, M.; Milke, R.; Kranz, C. \& Neusser, G. (2017). Silica nanoparticle aggregation in calcite replacement reactions. Scientific Reports, 7(1): 1-6. https:// doi.org/10.1038/s41598-017-06458-8

Maliva, R. G. \& Siever, R. (1989). Chertification histories of some Late Mesozoic and Middle Palaeozoic platform carbonates. Sedimentology, 36: 907-926. https://doi.org/10.1111/j.1365-3091.1989.tb01753.x

Martínez del Olmo, W. (2019). Cambio climático, acuerdos de París y trampas geológicas donde secuestrar el CO2 en España. Revista de la Sociedad Geológica de España, 32: 87-106.

Meyers, W. J. (1977). Chertification in the Mississippian Lake Valley Formation, Sacramento Mountains, New Mexico. Sedimentology, 24: 75-105. https:// doi.org/10.1111/j.1365-3091.1977.tb00121.x

Pineda, A.; Giner J.; Zazo C. \& Goy J.L. (1981). Mapa Geológico de España 1.50.000, hoja no 1046 (Carboneras) y memoria. IGME, Madrid 79 pp. 